\title{
METIL-FORMAMIDA NA CRIOPRESERVAÇÃO DE SÊMEN OVINO
}

\author{
CARlos Pereira das GraÇAS ${ }^{1}$, AleXandre In PiaO Gomes LiM ${ }^{2}$, ANdrei ANTONiOni Guedes \\ FIDELIS $^{3}$, JÚlio ROQUETE CARDOSO ${ }^{4}$, HÉlio BLUME $^{5}$, RAFAEL GIANELla MONDADORI ${ }^{6}$ \\ ${ }^{1}$ Médico Veterinário, Mestre, Empresa de Assistencia Tecnica e Extensão Rural, Brasília, DF, Brasil \\ ${ }^{2}$ Médico Veterinário Autônomo, Brasília, DF, Brasil \\ ${ }^{3}$ Médico Veterinário Autônomo, Uberlândia, MG, Brasil \\ ${ }^{4}$ Professor Doutor da Universidade Federal de Goiás, Goiânia, GO, Brasil. juliorcardoso@gmail.com \\ ${ }^{5}$ Professor Doutor da UPIS Faculdades Integradas, Brasília, DF, Brasil \\ ${ }^{6}$ Professor Doutor da Universidade Federal de Pelotas, Pelotas, RS, Brasil
}

\begin{abstract}
Objetivou-se avaliar a eficácia da metil-formamida na criopreservação do sêmen ovino. O pool de sêmen utilizado no experimento foi obtido a partir da coleta, com vagina artificial, do sêmen de quatro carneiros mestiços Santa Inês, com idade aproximada de quatro anos. As coletas foram realizadas uma vez por semana, por seis semanas consecutivas, correspondendo, cada semana, a uma repetição do experimento. As frações do pool foram diluídas em cinco diferentes meios de congelação: (1) trisgema com 5,3\% de glicerol (TG5,3G); (2) tris-gema com $3 \%$ de metil-formamida (TG3MF); (3) tris-gema com 5\% de metilformamida (TG5MF); (4) tris-gema com 7\% de metil-formamida (TG7MF); (5) tris-gema com 9\% de metil-formamida (TG9MF). Foram avaliadas a motilidade progressiva e o vigor das células espermáticas e realizado o teste de termorresistência pós-descongelação. O
\end{abstract}

tratamento que obteve maior motilidade foi o TG5,3G (50\%), seguido do TG3MF (38\%) e os tratamentos que apresentaram menor motilidade progressiva foram TG5MF (29\%), TG7MF (1,0\%), TG9MF $(6,0 \%)$. Os meios contendo metil-formamida apresentaram resultados inferiores ao meio controle para preservar a integridade morfológica dos espermatozoides, sendo que nos meios TG7MF e TG9MF menos de 60\% de espermatozóides apresentaram-se morfologicamente normais. Os espermatozoides do meio TG5,3G apresentaram motilidade $(15 \%)$ e vigor $(2,8)$ similares aos do meio TG3MF (15\% e 2,6, respectivamente) no teste de termorresistência, mas o meio TG5,3G preservou melhor a integridade funcional da membrana plasmática. $\mathrm{O}$ glicerol foi mais eficiente como crioprotetor do que a metilformamida na criopreservação de sêmen ovino.

PALAVRAS-CHAVE: Amidas; carneiros; glicerol; tris-gema.

\section{METHYL-FORMAMIDE IN RAM SEMEN CRYOPRESERVATION}

\section{ABSTRACT}

The aim of this study was to evaluate the efficacy of methyl-formamide in ram semen cryopreservation. The semen pool used in this experiment was obtained by artificial vagina collection from four mixed breed Santa Inês rams, around four years of age. Semen collection was performed once a week, during six weeks. Each week corresponded to one experiment replication. The semen pool was divided in five fractions in order to be diluted in one of the following freezing media: (1) tris-egg yolk with $5.3 \%$ of glycerol (TG5.3G); (2) tris-egg yolk with $3 \%$ of methyl-formamide (TG3MF); tris-egg yolk with $5 \%$ of methyl-formamide (TG5MF); tris-egg yolk with $7 \%$ of 
methyl-formamide (TG7MF); tris-egg yolk with 9\% of methyl-formamide (TG9MF). Semen progressive motility, vigor and thermoresistance were evaluated. The treatments TG5.3G (50\%) and TG3MF (38\%) showed higher progressive motility after thawing, while TG5MF (29\%), TG7MF (1\%) and TG9MF (6\%) showerd lower motility. Freezing media containing methyl-formamide were less effective in preserving spermatozoa membrane integrity and morphology than control media. In TG7MF

KEYWORDS: Amide; glycerol; ram; tris-egg yolk.

\section{INTRODUÇÃO}

Vários diluentes e técnicas têm sido desenvolvidos e adaptados com o objetivo de melhorar a criopreservação de sêmen ovino, mas a fertilidade após inseminação artificial cervical com sêmen congelado permanece significativamente menor do que a obtida com sêmen fresco (O'HARA et al., 2010, CÂMARA \& GUERRA, 2011). Isso pode ser decorrente de alterações bioquímicas e moleculares espermáticas ocorridas durante o processo de congelação e descongelação, redução do tempo de sobrevivência e falha de transporte após deposição no sistema genital da ovelha (MORAES et al., 1998).

Assim sendo, há a necessidade de investimentos em pesquisas a fim de se aumentar a eficiência dos meios atuais de criopreservação do sêmen. A melhoria da qualidade do sêmen ovino congelado pode levar ao aumento dos índices de nascimento de ovelhas inseminadas pela via cervical e, como essa técnica torna o emprego do sêmen mais acessível nesta espécie, isto contribuiria para reduzir os custos e a necessidade de mão-de-obra especializada requeridas pela inseminação intrauterina utilizando a laparoscopia (MORAES et al., 1998).

Tanto o glicerol quanto as amidas são crioprotetores permeantes, porém as amidas apresentam menor viscosidade e menor peso molecular do que o glicerol (metil formamida, 59; dimetil-formamida, 73; glicerol, 92). Portanto, essas características podem resultar em uma maior permeabilidade da membrana plasmática a esses agentes, gerando menores danos osmóticos aos espermatozóides (ALVARENGA et al., 2005).

Há poucos trabalhos, porém com resultados favoráveis, acerca do uso de amidas como crioprotetores para o sêmen de algumas espécies, como coelhos (OKUDA et al., 2007), cães (FUTINO et al., 2010) e equinos and TG9MF extenders, less than 60\% spermatozoa showed normal morphology. After thermoresistance test, semen cryopreserved in TG3MF showed vigor (2.6) and motility (15\%) statistically similar to TG5.3G media (15\% and 2.8, respectively); however, the extender TG5.3G was more effective in preserving plasma membrane functional integrity. In conclusion, in the experimental conditions used, glycerol showed more cryoprotectant potential than methyl-formamide.

(ALVARENGA et al., 2005). De forma geral, os resultados não apontam grandes diferenças em relação ao emprego do glicerol nos parâmetros avaliados in vitro; entretanto, ensaios de fertilidade têm demonstrado a maior eficiência in vivo das amidas na espécie equina (VIDAMENT et al., 2002; SQUIRES et al., 2004), inclusive em garanhões com sêmen de baixa congelabilidade (ALVARENGA et al., 2005). Dentre as amidas, somente a dimetilformamida foi testada em pequenos ruminantes e os resultados não apontaram benefício dessa amida em detrimento ao glicerol para o congelamento de sêmen, tanto de caprinos (SILVA et al., 2006; BEZERRA et al., 2011), quanto de ovinos (MOUSTACAS et al., 2011). Este, portanto, até onde pudemos constatar, é o primeiro estudo com a utilização da metilformamida na cripopreservação de sêmen ovino.

Portanto, pretendeu-se neste experimento testar alternativas para otimizar o processo de criopreservação, permitindo a utilização em maior escala da inseminação artificial com sêmen congelado em ovinos. Assim, objetivou-se testar in vitro a eficiência da metil-formamida em diferentes concentrações $(3 \%, 5 \%, 7 \%$ e $9 \%)$ na criopreservação do sêmen ovino, utilizando como controle o glicerol.

\section{MATERIAL E MÉTODOS}

Utilizaram-se como doadores de sêmen quatro carneiros mestiços Santa Inês com idade aproximada de 4 anos e peso em torno de $60 \mathrm{~kg}$, provenientes da Fazenda Lagoa Bonita - Campus II das Faculdades Integradas UPIS, localizada em Planaltina-DF.

Os animais foram mantidos em regime semi-extensivo de criação e permaneceram em pastagem de tifton (Cynodon spp), suplementados com mistura mineral capri-ovis INTEGRAL $810^{\circledR}$ e receberam, como concentrado, ração com $20 \%$ 
de proteína bruta (Ração Comigo Ovino Corte $20^{\circledR}$, Rações Comigo, Rio Verde - GO) e água $a d$ libitum.

Os animais foram submetidos a uma coleta de sêmen semanal (durante seis semanas), perfazendo um total de seis amostras por animal. Cada coleta correspondeu a uma repetição do ensaio. Para tanto, utilizou-se uma vagina artificial $\left(\right.$ Walmur $^{\circledR}$ ) preenchida com água aquecida entre $42^{\circ} \mathrm{C}$ e $44^{\circ} \mathrm{C}$ e uma fêmea mestiça, com idade aproximada de cinco anos, como "manequim". Utilizaram-se somente os ejaculados com os seguintes padrões mínimos: $0,5 \mathrm{~mL}$ de volume, $70 \%$ de motilidade progressiva e vigor 3. Após as coletas, os tubos contendo sêmen foram acondicionados em uma caixa térmica com água aquecida a $30^{\circ} \mathrm{C}$ e conduzidos ao laboratório de reprodução para as análises. O sêmen foi colocado em banho-maria à temperatura de $30^{\circ} \mathrm{C}$ e mantido nessa temperatura durante todo o processo de avaliação do vigor e motilidade. As quatro amostras dos ejaculados foram misturadas formando um pool a ser utilizado nas etapas posteriores.

A motilidade progressiva e o vigor do sêmen foram avaliados em cinco momentos distintos: individualmente logo após a coleta, após a formação do pool, após a diluição e resfriamento, após a descongelação e após o teste de termorresistência. Para avaliação microscópica, uma aliquota de $5 \mu \mathrm{L}$ de sêmen fresco foi colocada entre lâmina e lamínula, previamente aquecidas em placa aquecedora a $37^{\circ} \mathrm{C}$, para avaliação em microscópio óptico (aumento de 100X e 400X) da motilidade espermática progressiva, expressa em percentagem ( 0 a 100), e do vigor espermático, numa escala de 0 (imobilidade) a 5 (rápida mobilidade).

As alterações morfológicas dos espermatozóides foram avaliadas em esfregaços corados com corante vermelho do congo e violeta de genciana, sendo observadas 200 células em microscópio óptico, utilizando-se objetiva de imersão (100X). Essa avaliação foi realizada em dois momentos: logo após a formação do pool e após a descongelação. A concentração espermática foi determinada em câmara hematimétrica (câmara de Neubauer).

A integridade funcional da membrana plasmática dos espermatozóides foi avaliada após o descongelamento por meio do teste hiposmótico (HO), utilizando-se $1 \mathrm{~mL}$ de solução hiposmótica de 100mOsmol/L (JEYANDREN et al., 1984), previamente aquecida a $37^{\circ} \mathrm{C}$, acrescida de $10 \mu \mathrm{L}$ de sêmen. Essa solução foi previamente incubada por uma hora em banho-maria a $37^{\circ} \mathrm{C}$ (FONSECA et al., 2001). Avaliaram-se 200 células e as mesmas foram classificadas quanto à presença ou não de cauda enrolada e o resultado foi determinado em porcentagem. As células que apresentaram cauda enrolada foram classificadas como células com membrana plasmática funcional.

$O$ teste de termorresistência lento foi realizado com a manutenção da amostra por 4 horas à temperatura de $37^{\circ} \mathrm{C}$. Para tanto, descongelou-se uma palheta de cada diluição, por 1 minuto a $37^{\circ} \mathrm{C}$ e adicionou seu conteúdo em tubos de $1,5 \mathrm{~mL}$ que foram mantidos em banhomaria a $37^{\circ} \mathrm{C}$. A avaliação da motilidade progressiva e vigor dessas amostras foi realizada a cada 60 minutos.

Depois da formação do pool, o sêmen foi dividido em cinco alíquotas de $500 \mu \mathrm{L}$ e a cada uma delas foi adicionado $1,5 \mathrm{~mL}$ dos diferentes meios de congelação. $\mathrm{O}$ meio base para $\mathrm{o}$ experimento foi o Tris-gema (SALAMON \& MAXWELL, 2000), variando o tipo e a concentração de crioprotetor. Foram feitas alíquotas dos meios no volume de utilização $(1,5 \mathrm{~mL})$, que foram armazenadas em freezer a $-40^{\circ} \mathrm{C}$. Antes de cada coleta de sêmen, um tubo de cada meio diluidor foi descongelado e pré-aquecido a $30^{\circ} \mathrm{C}$. Os grupos experimentais testados foram os seguintes: (1) Trisgema com 5,3\% de glicerol (Controle); (2) Trisgema com 3\% de metil-formamida (MF3); (3) Trisgema com 5\% de metil-formamida (MF5); (4) Trisgema com 7\% de metil-formamida (MF7); e (5) Trisgema com $9 \%$ de metil-formamida (MF9).

$\mathrm{O}$ sêmen diluído foi resfriado até $4^{\circ} \mathrm{C}$ por 50 minutos, seguido por um período de 30 minutos de estabilização. O congelamento se deu em vapor de nitrogênio. As palhetas foram colocadas em um suporte flutuante $(21,5 \mathrm{~cm}$ de comprimento e 15 $\mathrm{cm}$ de largura), ajustado para que elas permanecessem a $5 \mathrm{~cm}$ de altura da superfície do nitrogênio líquido. Esse procedimento foi realizado em uma caixa térmica $(28 \mathrm{~cm}$ de altura, $20 \mathrm{~cm}$ de largura e $23 \mathrm{~cm}$ de comprimento) por 20 minutos (de $4{ }^{\circ} \mathrm{C}$ até $\left.-80{ }^{\circ} \mathrm{C}\right)$. O procedimento para congelação foi avaliado por um termômetro digital (GTH $215^{\circledR}$, Greisinger Electronic, $\mathrm{GmbH}$ ). Concluída essa etapa do processo, as palhetas foram imersas no nitrogênio líquido, onde ficaram armazenadas por pelo menos uma semana antes do descongelamento e avaliações in vitro previamente descritas. 
Cada coleta de sêmen correspondeu a uma repetição do ensaio. Os resultados de motilidade progressiva, vigor, patologia espermática e integridade de membrana foram transformados por arco seno da raiz quadrada para reduzir o coeficiente de variação e avaliados, dentro dos diferentes momentos de processamento, por análise de variância. A comparação entre as médias foi realizada pelo teste de Duncan. O programa utilizado para as análises foi o SAS System. Foram consideradas diferentes análises com $\mathrm{p}<0,05$.

\section{RESULTADOS E DISCUSSÃO}

O pool de sêmen fresco apresentou valores médios de motilidade progressiva de $89,1 \%$, vigor de 5,0 e percentagem de espermatozóides morfologicamente normais de $84,66 \%$. Esses parâmetros demonstram que o sêmen era de boa qualidade e esses índices se enquadravam nos requisitos mínimos para congelação, conforme o CBRA. Após a diluição nos meios crioprotetores, os espermatozóides dos meios TG5MF e TG7MF já apresentaram motilidade progressiva inferior $(\mathrm{p}<0,05)$ ao controle $(\mathrm{TG}, 3 \mathrm{G})$. O vigor também foi estatisticamente menor $(\mathrm{p}<0,05)$ no grupo TG5MF (Tabela 1), demonstrando que a metilformamida (MF), nessas concentrações, reduziu a atividade espermática. Por outro lado, as amostras avaliadas no período pós-resfriamento não apresentaram diferenças $(\mathrm{p}>0,05)$ quanto a esses parâmetros, sugerindo que os efeitos deletérios anteriores foram transitórios, sendo a atividade espermática restabelecida durante o período de resfriamento do sêmen, ou seja, após o equilíbrio entre a célula e meio crioprotetor.

Tabela 1. Motilidade progressiva (MP) e vigor do sêmen ovino criopreservado em meios diluidores contendo diferentes concentrações de metil-formamida nos diferentes estágios de avaliação dos tratamentos

\begin{tabular}{lcccccc}
\hline & \multicolumn{2}{c}{ Pós-diluição } & \multicolumn{2}{c}{ Pós-resfriamento } & \multicolumn{2}{c}{ Pós-descongelação } \\
\cline { 2 - 7 } & MP & Vigor & MP & Vigor & MP & Vigor \\
\hline TG5,3G & $86,6^{\mathrm{a}} \pm 4,0$ & $4,8^{\mathrm{a}} \pm 0,4$ & $79,0^{\mathrm{a}} \pm 6,5$ & $3,8^{\mathrm{a}} \pm 0,6$ & $50,0^{\mathrm{a}} \pm 18,7$ & $4,0^{\mathrm{a}} \pm 0,7$ \\
TG3MF & $84,1^{\mathrm{ab}} \pm 3,7$ & $4,5^{\mathrm{ab}} \pm 0,8$ & $76,0^{\mathrm{a}} \pm 4,2$ & $3,6^{\mathrm{a}} \pm 0,5$ & $38,0^{\mathrm{ab}} \pm 8,3$ & $3,2^{\mathrm{a}} \pm 0,4$ \\
TG5MF & $80,0^{\mathrm{b}} \pm 4,5$ & $4,0^{\mathrm{b}} \pm 0,6$ & $73,0^{\mathrm{a}} \pm 6,7$ & $3,2^{\mathrm{a}} \pm 0,4$ & $29,0^{\mathrm{b}} \pm 11,4$ & $3,4^{\mathrm{a}} \pm 0,8$ \\
TG7MF & $79,1^{\mathrm{b}} \pm 5,8$ & $4,1^{\mathrm{ab}} 0,4$ & $72,0^{\mathrm{a}} \pm 7,6$ & $3,2^{\mathrm{a}} \pm 0,4$ & $1,0^{\mathrm{c}} \pm 2,2$ & $0,4^{\mathrm{b}} \pm 0,9$ \\
TG9MF & $81,6^{\mathrm{ab}} \pm 4,0$ & $4,1^{\mathrm{ab}} \pm 0,4$ & $72,0^{\mathrm{a}} \pm 9,1$ & $3,4^{\mathrm{a}} \pm 0,5$ & $6,0^{\mathrm{c}} \pm 10,8$ & $1,0^{\mathrm{b}} \pm 1,7$ \\
\hline
\end{tabular}

TG5,3G - Tris-gema com 5,3\% de glicerol (controle); TG3MF, TG5MF, TG7MF e TG9MF - Tris-gema com 3\%, 5\%, 7\% e 9\% de metil-formamida, respectivamente. Letras diferentes na mesma coluna diferem entre si $(\mathrm{p}<0,05)$. Os valores estão como média \pm desvio padrão.

Conforme demonstrado na Tabela 1, após o descongelamento do sêmen, somente o meio contendo metil-formamida na concentração de $3 \%$ (TG3MF) foi capaz de proteger os espermatozóides das injúrias do processo de criopreservação, com valores da motilidade progressiva e vigor similares aos apresentados pelo sêmen criopreservado com o glicerol (TG5,3G). Esses resultados coincidem com os obtidos com sêmen de cão congelado com MF (FUTINO et al., 2010). Resultados de estudos utilizando amidas em algumas espécies apontam para um melhor desempenho da MF em detrimento à dimetilformamida (DMF), embora haja poucos trabalhos com o uso da MF. Nesse aspecto, tanto neste estudo, com ovinos, quanto em estudos com cães (FUTINO et al., 2010), a MF foi capaz de preservar a motilidade e o vigor dos espermatozoides após o descongelamento. Já o emprego de DMF gerou índices de motilidade inferiores aos obtidos com o uso do glicerol em cães (LOPES et al., 2009;
FUTINO et al., 2010; MOTA FILHO et al., 2011); em suínos (BURANAAMNUAY et al., 2011; MALO et al., 2012); em ovinos (MOUSTACAS et al., 2011) e em caprinos (BEZERRA et al., 2011).

É importante ressaltar que os tratamentos com maiores concentrações de metil-formamida (7 e 9\%) apresentaram níveis muito baixos de motilidade pós-descongelamento, o que inviabiliza esforços posteriores para estudos de meios diluidores utilizando esse crioprotetor em níveis superiores a $5 \%$ em ovinos, demonstrando também a toxicidade desses componentes a 7 e $9 \%$ no meio de congelamento de sêmen ovino.

Assim como observado neste estudo, a utilização das amidas para criopreservação de sêmen de garanhões com boa congelabilidade não proporcionou aumento da motilidade e sim resultados semelhantes aos obtidos pelo uso do glicerol (VIDAMENT et al., 2002; SQUIRES et al., 2004; ALVARENGA et al., 2005); todavia, a 
utilização desses agentes em garanhões com baixa resistência ao processo de criopreservação resultou em índices de motilidade progressiva e de fertilidade significativamente melhores do que os proporcionados pelo glicerol (GOMES et al., 2002; ALVARENGA et al., 2002; ALVARENGA et al., 2005). Diferentemente da espécie equina, não há até o presente momento dados sobre taxa de fertilidade de pequenos ruminantes em inseminação artificial com o uso de amidas como crioprotetores.

Quanto ao teste de termorresistência, o protocolo preconizado pelo CBRA para a espécie ovina determina que o sêmen seja considerado apto se a amostra contiver pelo menos $30 \%$ dos espermatozóides com motilidade progressiva. Neste estudo, não houve diferença estatística $(p>0,05)$ entre os resultados de motilidade e vigor após o teste de termorresistência entre o meio controle (TG5,3G) e o meio com 3\% de metil-formamida (TG3MF), com ambas as amostras apresentando $15 \%$ de motilidade progressiva, nível insatisfatório de acordo com o preconizado pelo CBRA. Os espermatozóides do meio TG5MF apresentaram motilidade progressiva baixa (6\%) e nos meios TG7MF e TG9MF não havia mais motilidade após o período de incubação do sêmen (Tabela 2). Esses resultados sustentam o que foi observado em cães, em que também não houve diferenças estatísticas entre os tratamentos com MF e glicerol (FUTINO et al., 2010). A DMF na concentração de 5\% também manteve a longevidade dos espermatozóides em níveis similares ao glicerol a 7\% na espécie caprina (SILVA et al., 2006); contudo, em cães, a DMF foi inferior à MF e ao glicerol (FUTINO et al., 2010).

Neste estudo, a integridade funcional da membrana plasmática avaliada pelo teste $\mathrm{HO}$ foi melhor preservada pelo glicerol $(44,4 \% \pm 8,6)$ do que pelas amidas. Entre os meios contendo metilformamida, as concentrações de 3 e $5 \%(33,6 \% \pm 7,1$ e $25,4 \% \pm 4,9$, respectivamente) foram inferiores estatisticamente ao meio contendo glicerol, mas não diferiram entre si. Os meios contendo 7 e $9 \%$ de metil-formamida $(13,8 \% \pm 9,4$ e $9,0 \% \pm 7,5$, respectivamente) apresentaram o menor número de espermatozóides reativos ao teste hiposmótico, sendo estatisticamente inferior $(p<0,05)$ aos demais grupos. Esses resultados sustentam os achados na literatura. O glicerol foi mais eficiente do que a MF e DMF na preservação da integridade da membrana plasmática de espermatozóides de cães (FUTINO et al., 2010) e melhor do que a DMF em espermatozóides de ovinos (MOUSTACAS et al., 2011). Já em caprinos, tanto a integridade estrutural, quanto a funcional foram melhor preservadas pela DMF do que pelo glicerol (BEZERRA et al., 2011).

Tabela 2. Motilidade progressiva (\%) e vigor após teste de termorresistência lento, de espermatozoides ovinos após congelação e descongelação em diferentes meios

\begin{tabular}{lcc}
\hline Tratamentos & Motilidade & Vigor \\
\hline TG5,3G & $15,0^{\mathrm{a}} \pm 7,0$ & $2,8^{\mathrm{a}} \pm 0,4$ \\
TG3MF & $15,0^{\mathrm{a}} \pm 7,0$ & $2,6^{\mathrm{a}} \pm 0,5$ \\
TG5MF & $6,0^{\mathrm{b}} \pm 4,2$ & $1,6^{\mathrm{b}} \pm 0,9$ \\
TG7MF & $0,0^{\mathrm{b}} \pm 0,0$ & $0,0^{\mathrm{c}} \pm 0,0$ \\
TG9MF & $0,0^{\mathrm{b}} \pm 0,0$ & $0,0^{\mathrm{c}} \pm 0,0$ \\
\hline
\end{tabular}

TG5,3G - Tris-gema com 5,3\% de glicerol (controle); TG3MF, TG5MF, TG7MF e TG9MF - Tris-gema com 3\%, 5\% , 7\% e 9\% de metil-formamida, respectivamente.

Letras diferentes na mesma coluna diferem entre si $(\mathrm{p}<0,05)$. Valores estão apresentados como média \pm desvio padrão. Média do pool de quatro carneiros (6coletas/carneiro)

Segundo OETTLÉ (1993), a morfologia espermática apresenta correlação com a fertilidade, sendo que a percentagem de espermatozóides normais menor que $60 \%$, diminui a fertilidade em $87 \%$. Neste estudo, os meios contendo metilformamida apresentaram resultados estatisticamente inferiores ao meio controle contendo glicerol em preservar a integridade morfológica dos espermatozóides, sendo que nos meios TG5MF e TG7MF menos de $60 \%$ de espermatozoides apresentaram-se morfologicamente normais (Tabela
3). Esses resultados contrariam os obtidos em cães, em que o meio contendo MF manteve melhor a morfologia espermática do que os meios contendo DMF e glicerol (FUTINO et al., 2010). No que se refere à DMF, tanto em cães (ZIMMERMANN et al., 2007; FUTINO et al., 2010), quanto em caprinos (BEZERRA et al., 2011), não foram observadas diferenças morfológicas estatisticamente significativas em relação às células do meio controle contendo glicerol. 
Tabela 3. Morfologia espermática (\%) de espermatozóides normais e com alterações de cabeça, peça intermediária (PI) e de cauda em sêmen de ovino criopreservados em meios diluidores contendo diferentes concentrações de metil-formamida

\begin{tabular}{lllll}
\hline Tratamentos & $\begin{array}{l}\text { Espermatozoides } \\
\text { normais }\end{array}$ & Patologia de cabeça & Patologia de PI & Patologia de cauda \\
\hline Sêmen fresco & $84,6 \pm 5,1$ & $8,0 \pm 4,9$ & $3,0 \pm 2,0$ & $4,3 \pm 2,6$ \\
TG5,3G & $84,2^{\mathrm{a}} \pm 2,1$ & $5,8^{\mathrm{b}} \pm 1,9$ & $3,6^{\mathrm{a}} \pm 1,5$ & $6,40^{\mathrm{b}} \pm 2,07$ \\
TG3MF & $76,8^{\mathrm{b}} \pm 3,1$ & $6,2^{\mathrm{b}} \pm 1,9$ & $6,2^{\mathrm{a}} \pm 3,5$ & $11,2^{\mathrm{b}} \pm 2,94$ \\
TG5MF & $66,4^{\mathrm{c}} \pm 4,6$ & $18,0^{\mathrm{a}} \pm 5,4$ & $6,2^{\mathrm{a}} \pm 1,5$ & $9,40^{\mathrm{b}} \pm 2,88$ \\
TG7MF & $56,4^{\mathrm{d}} \pm 5,3$ & $16,0^{\mathrm{a}} \pm 4,8$ & $7,2^{\mathrm{a}} \pm 1,6$ & $16,8^{\mathrm{b}} \pm 7,2$ \\
TG9MF & $46,4^{\mathrm{e}} \pm 7,1$ & $18,4^{\mathrm{a}} 5,4$ & $7,8^{\mathrm{a}} \pm 8,5$ & $27,9^{\mathrm{a}} \pm 7,8$ \\
\hline
\end{tabular}

TG5,3G - Tris-gema com 5,3\% de glicerol (controle); TG3MF, TG5MF, TG7MF e TG9MF - Tris-gema com 3\%, 5\% , 7\% e 9\% de metil-formamida respectivamente. Letras diferentes na mesma coluna diferem entre si $(\mathrm{p}<0,05)$. Valores estão apresentados como média \pm desvio padrão. Média do pool de quatro carneiros (6 coletas/carneiro)

BEZERRA et al. (2011) apontam que a DMF não é tão eficiente como crioprotetor para espermatozóides de ovinos como é para os de caprinos, caninos, suínos e equinos e que essas variações podem estar envolvidas com diferenças estruturais nas membranas das células espermáticas entre as espécies. Neste estudo, entretanto, observouse que, diferente da DMF, os resultados obtidos com a MF em concentrações menores que 5\% se equiparam aos do glicerol na maior parte dos parâmetros avaliados, sugerindo que o menor peso molecular da MF em relação à DMF também pode ter implicações nessas diferenças de resultados entre as amidas na espécie ovina. Por isso, sugerimos novas pesquisas com metil-formamida em concentrações menores do que $5 \%$ e, em especial, ensaios de fertilidade usando o sêmen preservado com esse composto.

\section{CONCLUSÕES}

O sêmen ovino pode ser congelado utilizando-se a metil-formamida como crioprotetor na concentração de $3 \%$

\section{AGRADECIMENTOS}

À Faculdades Integradas UPIS, pela disponibilização de suas instalações, do material de laboratório e dos animais utilizados neste estudo.

\section{REFERÊNCIAS}

ALVARENGA, M.A.; LEÃO, K.M.; PAPA, F.O. Improvement of stallion semen post-thaw motility with utilization of dimethylformamide as cryoprotector. Theriogenology, v.57, n.1, p.459, 2002.

ALVARENGA, M.A.; PAPA, F.O.; LANDIMALVARENGA, F.C.; MEDEIROS, A.SL. Amides as cryoprotectants for freezing stallion semen: A review. Animal Reproduction Science, v.89, n.1-4, p.105-113, 2005.

BEZERRA, F. S. B.; CASTELO, T. S.; ALVES, H. M.; OLIVEIRA, I.R.S.; LIMA, GL.; PEIXOTO, G. C. X.; BEZERRA, A. C. S. D; SILVA, A. R. Objective assessment of the cryoprotective effects of dimethylformamide for freezing goat semen. Cryobiology, v. 63, p. 263-266, 2011.

BURANAAMNUAY, K.; GROSSFELD, R.; STRUCKMANN, C.; RATH, D. Influence of cryoprotectants glycerol and amides, combined with antioxidants on quality of frozen-thawed boar sperm. Animal Reproduction Science, v. 127, n. 1-2, p. 56-61, 2011.

CÂMARA, D. R.; GUERRA, M. M. P. Refrigeração e criopreservação do sêmen ovino: danos inerentes à técnica e influência da suplementação do meio com antioxidantes sobre a qualidade espermática. Revista Brasileira de Reprodução Animal, v.35, n.1, p.33-40, 2011.

CBRA - COLEGIO BRASILEIRO DE REPRODUÇÃO ANIMAL, Manual para Exame Andrológico e Avaliação de sêmen Animal (2ed.). Belo Horizonte, CBRA, 1998, 49p. Disponível em http://pt.scribd.com/doc/151892128/Manual-para-ExameAndrologico-e-Avaliacao-de-Semen-Animal, Acesso em 21 Out 2013.

SALAMON, S.; MAXWELL, W.M.C. Storage of ram semen. Animal Reproduction Science. v. 62, n. 1-3, p. 77-111, 2000.

FUTINO, D.O.; MENDES, M.C.B.; MATOS, W.N.L.; MONDADORI, R.G.; LUCCI, C.M. Glycerol, MethylFormamide and Dimethyl-Formamide in Canine Semen Cryopreservation. Reproduction in Domestic Animals. v.45, n. 2, p. 214-220, 2010.

GOMES, G.M.; JACOB, J.F.C.; MEDEIROS, A.S.L. Improvement of stallion spermatozoa preservation with alternative cryoprotectans for the Mangalarga Marchador breed. Theriogenology, v. 58, p. 277-279, 2002. 
JEYENDRAN, R.S.; VANDER-VEN, H.H.; PEREZPELAEZ, M.; CRABO, B.G.; L. ZANEVLD, J.D. Development of an assay to assess the functional integrity of the human sperm membrane and its relationship to other semen characters. Journal of Reproduction and Fertility, v. 70, p. 219-228, 1984.

LOPES, K.R.; COSTA, L.L.; LIMA, G.L.; SOUZA, A.L.; SILVA, A.R. Dimethylformamide is no better than glycerol for cryopreservation of canine semen. Theriogenology, v. 72, n. 5, p.650-4, 2009.

MALO, C.; GIL, L.; CANO, R.; MARTÍNEZ, F.; GARCÍA, A.; JEREZ, R. A. Dimethylformamide is not better than glycerol for cryopreservation of boar semen. Andrologia, v.44, Suppl 1, p. 605-10, 2012

MEDEIROS, A.S.L.; GOMES, G.M.; CARMO, M.T.; PAPA, F.O.; ALVARENGA, M.A. Cryopreservation of stallion sperm using different amides. Theriogenology, v.58, p.273-276, 2002.

MORAES, C.N.; NEVES, J.P.; GONÇALVES, P.B.D.; OLIVEIRA, J.F.C.; SCHWEITZER, C.M. Criopreservação do sêmen ovino em Pellets com etileno glycol. Ciência Rural, v.28, n.2,p.287-292 1998.

MOTA FILHO, A.C.; TELES, C.H.; JUCÁ, R.P.; CARDOSO, J.F.; UCHOA, D.C.; CAMPELLO, C.C.; SILVA, A.R.; SILVA, L.D. Dimethylformamide as a cryoprotectant for canine semen diluted and frozen in ACP-106C. Theriogenology. v.76, n.7, p.1367-72, 2011.

MOUSTACAS, V.S.; CRUZ, B.C.; VARAGO, F.C.; MIRANDA, D.A.; LAGE, P.G.; HENRY, M. Extenders containing dimethylformamide associated or not with glycerol are ineffective for ovine sperm cryopreservation.
Reproduction in Domestic Animals, v.46, p.924-925, 2011.

O'HARA, L., HANRAHAN, J.P., RICHARDSON, L., DONOVAN, A., FAIR, S., EVANS, A.C.O., LONERGAN, P. Effect of storage duration, storage temperature, and diluent on the viability and fertility of fresh ram sperm. Theriogenology, v.73, p.541-549, 2010.

OKUDA, Y.; SEITA, Y.; HISAMATSU, S.; SONOKI, S.; SHINO, M.; MASAOKA, T.; INOMATA, T.; KAMIJO, S.I.; KASHIWAZAKI, N. Fertility of spermatozoa cryopreserved with $2 \%$ acetamide or glycerol through artificial insemination in the Japanese White Rabbit, Experimental Animals, v.56, p.29-34, 2007.

SILVA, A.F.; COSTA, E.P.; OLIVEIRA, F.A.; TORRES, C.A.A.; HASS, G.T.S.; NASCIMENTO, V. A. Uso de dimetil-formamida associada ou não ao glicerol na criopreservacao de semen caprino. Revista Brasileira de Zootecnia, v.35, p.452-456, 2006.

SQUIRES, E.L.; KEITH S.L.; GRAHAM, J.K. Evaluation of alternative cryoprotectants for preserving stallion spermatozoa. Theriogenology, v.62, n.6, p.1056-65, 2004.

VIDAMENT, M.; DAIRE, J.M.; YVON, J.M.; DOLIGEZ, P.; BRUNEAU, B.; MAGISTRINI, M.; ECOT, P. Motility and fertility of stallion semen frozen with glycerol and/or dimethyl formamide. Theriogenology, v.58, p.1-3, 2002.

ZIMMERMANN, M; SANTOS, T.E.; FIDELIS, A.A.G.; BLUME, H.; MONDADORI, R.G. Uso de dimetilformamida e água de coco na criopreservação de sêmen canino. Bioscience Journal, v.23, n.1, p. 96-100, 2007. 